\title{
A study the various clinical and electrophysiological parameters of severity of carpal tunnel syndrome, their correlation with post-operative recovery
}

\author{
Sushil Ramesh Nehete, Binita B. Raut, Amita S. Hiremath, R. Mukund Thatte \\ Department of Plastic Surgery, Bombay Hospital and Institute of Medical Sciences, Mumbai, Maharashtra, India
}

Address for correspondence: Dr. R. Mukund Thatte, 402, Vimal Smriti, Dr. Ghanti Road, Dadar East, Mumbai - 400 014, Maharashtra, India. E-mail: mthatte@gmail.com

\section{ABSTRACT}

Objective: We aimed to study the various clinical and electrophysiological parameters of severity of carpal tunnel syndrome (CTS) and to see if the severity of CTS affects recovery after surgery. Patients and Methods: A prospective study of 35 patients suffering from CTS. Clinical severity was assessed using visual analogue scale and standard questionnaires such as Levine and Disabilities of Arm, Shoulder and Hand questionnaires. All the patients underwent electrophysiological evaluation to assess electrophysiological severity of CTS. According to modified Padua classification, they were classified into three groups, namely, minimal to mild, moderate and severe to extreme. All patients underwent Carpal tunnel release in our unit. The clinical assessment was repeated 3 months post-operatively. Results: Out of 33 patients, majority $(65.7 \%)$ of the patients were suffering from moderately severe CTS. The clinical provocative tests were positive in majority of patients. Clinically and statistically significant $(P<0.001)$ improvement was seen in all clinical severity scores. However, it did not show any statistical correlation with electrophysiological severity of the disease when compared among the groups. There was no association of age, gender of the patient, body mass index, hand dominance, affected side of the patient, results of provocative tests and the presence or absence of thenar muscle atrophy when compared among the three severity groups $(P>0.05)$. Conclusions: Although pre-operative clinical scores of severity and electrophysiology have a diagnostic role in CTS, they do not correlate with post-operative recovery and in turn fail to predict the extent of post-operative recovery before surgery.

\section{KEY WORDS}

Carpal tunnel syndrome; disabilities of arm; shoulder and hand score; electromyography nerve conduction velocity; Levine score

\begin{tabular}{|l|l|}
\hline \multicolumn{2}{|c|}{ Access this article online } \\
\hline Quick Response Code: & Website: \\
& www.ijps.org \\
\cline { 2 - 2 } & Dol: \\
\hline
\end{tabular}

This is an open access article distributed under the terms of the Creative Commons Attribution-NonCommercial-ShareAlike 3.0 License, which allows others to remix, tweak, and build upon the work non-commercially, as long as the author is credited and the new creations are licensed under the identical terms.

For reprints contact: reprints@medknow.com

How to cite this article: Nehete SR, Raut BB, Hiremath AS, Thatte RM. A study the various clinical and electrophysiological parameters of severity of carpal tunnel syndrome, their correlation with post-operative recovery. Indian J Plast Surg 2017;50:260-5. 


\section{INTRODUCTION}

arpal tunnel syndrome (CTS) is an important cause of pain, neurological symptoms and functional limitation of the hand. In fact, it is the most common cause of nerve compression of upper extremity according to data from the USA..$^{[1]}$ In the large majority of cases, the condition is idiopathic. CTS has a significant impact on individual well-being and economic activity.

Despite many scientific papers and publications, there is no unanimity regarding diagnostic criteria for CTS. Usually clinical symptoms and physical signs are helpful in making provisional diagnosis. Person-to-person variability in severity of signs and symptoms suggestive of CTS makes it more difficult to diagnose and manage patients suffering with CTS. Levine et al. have developed a self-administered questionnaire for assessing the severity of the CTS symptoms. ${ }^{[2]}$

Besides history and clinical examination, electrophysiological examination is the only reliable means of confirming CTS. Electrophysiological examination is highly specific and is reasonably sensitive in diagnosing CTS. ${ }^{[3,4]}$ It helps rule out other neuropathic and co-morbid conditions as a cause of the signs and symptoms seen in the patient.

This study was undertaken to analyse the relationship of clinical severity within various modified Padua's groups of electrophysiological severity of CTS and its impact on post-operative recovery and relief. This study is an effort to enhance our knowledge for categorising patients depending on the severity of CTS so that we can form clinical guidelines which will be helpful for us in predicting the extent of post-operative recovery and relief.

\section{PATIENTS AND METHODS}

All patients diagnosed with CTS and fulfilling inclusion and exclusion criteria (as below) were included in the study. The present study was a prospective study, which included 35 patients ( 31 females and 4 males). Patients presenting to the Department of Hand and Plastic surgery with typical hand symptoms such as pain, tingling and numbness and nocturnal sleep disturbance with paraesthesia were selected for the study after providing informed written consent.
The clinical examination of the patients consisted of history, physical examination and specific provocative tests on the hand. Baseline data such as name, age and gender were recorded for all the patients included in the study. Height and weight of all patients were also noted down. Body mass index (BMI) was calculated for all patients. The presence of co-morbid conditions such as diabetes mellitus and hypothyroidism was noted. The patient was also physically examined for various findings supporting the diagnosis of CTS. Thenar muscle atrophy was looked for. Provocative tests such as Phalen's sign, Tinel's signs and Durkan's tests were elicited and findings were noted down. Median nerve innervated area was examined for sensory evaluation and condition of the skin. The patient was examined to rule out other causes of hand symptoms such as cervical spondylosis or generalised diabetic neuropathy. Objective assessment of the motor power of median nerve was done by recording grip strength. A special note was taken about duration of symptoms, whether symptoms were present in one or both hands, handedness and profession of the patient. A visual analogue scale ${ }^{5}$ was used to make a subjective assessment of the patient's complaints. This is in Figure 1. A self-administered questionnaire formed by Levine et al. ${ }^{[2]}$ was introduced to obtain symptom severity score (SSS) and functional status score (FSS). Disabilities of arm, shoulder and hand (DASH) questionnaire ${ }^{[6]}$ was also used for patient's assessment.

Electrophysiological evaluation of each patient was done pre-operatively, from the Department of Neurophysiology in our hospital to avoid inconsistency in comparing values attributed to machine or operator. Readings of sensory nerve action potential (SNAP) (in $\mu \mathrm{v})$, peak amplitude of compound muscle action potential (in $\mathrm{mV}$ ), peak latency of SNAP (in ms) and conduction velocity of the SNAP (in $\mathrm{m} / \mathrm{s}$ ) were recorded. According to Padua's classification, ${ }^{[7]}$ patients were divided into five groups, namely, minimal, mild, moderate, severe and extremely severe CTS. For statistical convenience and significance, we modified this classification into three groups, namely, minimal to mild (Group I), moderate (Group II) and severe to extreme (Group III).

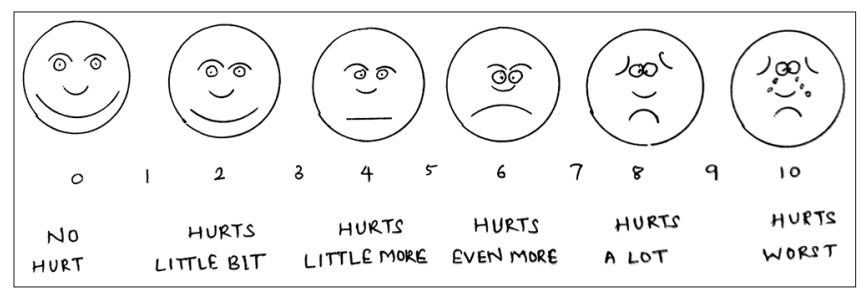

Figure 1: Visual analogue scale ${ }^{5}$ 
Except for electromyography (EMG) nerve conduction velocity (NCV) the whole assessment was repeated at follow-up at 3 months post-operatively. EMG NCV was not done at 3 months as it does not reliably show a change so quickly. The exact timing of improvement is not documented clearly, but it can take as long as 42 weeks for sensory latencies. ${ }^{[8]}$ Hence, EMG NCV may not be a reliable indicator of nerve recovery if done at 3 months when we had called all our patients for a review.

\section{Inclusion criteria}

1. All patients presenting to the Department of Plastic Surgery in our institute with hand symptoms such as pain, tingling and numbness and nocturnal sleep disturbance with paraesthesia

2. All patients who were diagnosed with CTS on electrophysiological studies.

\section{Exclusion criteria}

1. Patients who refused consent to take part in study

2. All patients with generalised neuropathies or double crush syndrome were excluded from the study.

\section{Variable and outcome measures}

To summarise, the following data of each patient were recorded for comparison before and after surgery

1. Visual analogue score (pre- and post-operative)

2. SSS (pre- and post-operative)

3. FSS (pre- and post-operative)

4. DASH score (pre- and post-operative).

These data were studied to analyse the correlation with the following electrophysiological variables of median nerve:

1. SNAP (in $\mu \mathrm{v}$ )

2. Peak latency of SNAP (in $\mathrm{mS}$ )

3. Conduction velocity of the SNAP (in $\mathrm{m} / \mathrm{s}$ )

4. Peak amplitude of compound muscle action potential (in $\mathrm{mV}$ )

5. Peak latency of motor nerve action potential (in $\mathrm{mS}$ ).

\section{Data analysis}

After data collection, data entry was done in Excel. Data analysis is done with the help of SPSS Software ver 15 and SigmaplotVer 11 (Systat Software Inc. San Jose, California, USA).

Quantitative data are presented with the help of mean, standard deviation (SD), median and interquartile range, comparison among study group is done with the help of Mann-Whitney test or unpaired $t$-test, intragroup comparison is done with the help of one-way analysis of variance or Kruskal-Wallis test as per results of normality test and multiple pair-wise comparison among group is done with Tukey's test or Holm-Sidak method.

Qualitative data are presented with the help of frequency and percentage table and association among study group is assessed with the help of Chi-square test.

$P<0.05$ is taken as significant level.

All patients were managed with surgical treatment. At our institute, we employ minimal invasive open technique for carpal tunnel release. ${ }^{[9]}$

\section{RESULTS}

In our study, majority of the patients were in the fourth, fifth and sixth decades of their life, and maximum 13 patients were in fifth decade with the mean age of 47.34 (SD 4.95) years and median of 47 years.

Thirty-three patients with 35 hands suffering from CTS were studied in the present study. It included 30 females and 3 males. Only one patient out of the 33 was left-hand dominant. Among the 35 affected hands, the right hand was affected 27 times (77.1\%). Almost 75\% (26) of patients were homemakers, whereas a few patients were artist, dentist or staff nurse who were required to perform skilled job and other patients were white-collar workers having clerical work, computer job, trader and teacher.

We classified our patients according to BMI. Patients with BMI 19-24 were considered to have appropriate weight for height. The groups were underweight (BMI <19), overweight (BMI 24-30), obese (BMI 30-40) and morbidly obese (BMI >40). Only 28.6\% (10) of the patients had appropriate weight for their height, whereas 20 of the 35 patients were overweight and 5 patients were obese. The Tinel's sign was positive in 27 (77.14\%) patients. Phalen's test was positive in $30(85.71 \%)$ out of 35 patients, whereas the Durkan's sign was positive in $30(85.71 \%)$ patients. On motor examination of all patients, $68.6 \%$ of patients were found to have thenar muscle atrophy on presentation.

According to modified Padua's classification, there were five patients who were falling in Group I of CTS, whereas 
23 and 7 patients were observed to be in Group II and III, respectively [Table 1].

The mean clinical severity scores were as shown in Table 2.

The mean values of various electrophysiological parameters studied with the SD in the modified Padua's groups were as shown in Table 3.

The change in the clinical severity score from pre-operative value to the post-operative value should indicate clinical improvement or deterioration. Hence, we compared the difference between pre-operative and post-operative scores (pre- and post-operative) in the three electrophysiological severity groups.

The difference between the pre- and post-operative scores was compared to analyse clinical post-operative recovery. All the scores showed statistically proved significant improvement on post-operative evaluation as shown in Table 4.

\section{DISCUSSION}

In the present study, we compared pre-operative clinical severity scores with their respective post-operative

Table 1: Distribution of patients according to modified Padua's classification

\begin{tabular}{lc}
\hline Electrophysiological severity & Frequency (\%) \\
\hline Group I & $5(14.3)$ \\
Group II & $23(65.7)$ \\
Group III & $7(20.0)$ \\
Total & $35(100.0)$ \\
\hline
\end{tabular}

Table 2: Clinical severity scores: Pre- and post-operative

\begin{tabular}{lcc}
\hline $\begin{array}{l}\text { Score (minimum- } \\
\text { maximum) }\end{array}$ & \multicolumn{2}{c}{ Mean (SD) } \\
\cline { 2 - 3 } VAS (0-10) & Pre-operative score & Post-operative score \\
SSS (0-44) & $2.29(1.58)$ & $0.94(1.55)$ \\
FSS (0-32) & $18.89(8.06)$ & $3.20(6.57)$ \\
DASH (30-150) & $82.89(19.31)$ & $2.00(4.52)$ \\
\hline VAS: Vis &
\end{tabular}

VAS: Visual analogue scale, SSS: Symptom severity score, FSS: Functional status score, DASH: Disabilities of arm, shoulder and hand, SD: Standard deviation values. We found that the clinical severity scores are statistically significantly improved after surgery [Table 5]. It indicates not only that surgery is the effective mode of treatment but also that the clinical severity scores, namely, visual analogue score, SSS, FSS and DASH score are very much predictive of the success of surgery and recovery of patient from the symptoms of CTS the patient was suffering from before surgery.

We also compared various electrophysiological parameters in the three severity groups to find that various electrophysiological parameters are effective in making diagnosis of CTS, especially when the patient is suffering from severe to extremely severe CTS.

You et al. ${ }^{[10]}$ studied the relationship between pre-operative clinical severity scales and abnormal electrodiagnostic measures in CTS patients. You et al. used the same Levine questionnaire which was also used in the present study. They found significant relationships between the clinical scales and nerve conduction measures. In the present study, we compared the difference between pre-operative and post-operative SSS which is obviously an indicator of recovery from the symptom complex the patient was suffering from.

On comparing the difference between pre-operative and post-operative SSSs among the three study groups, we have made depending on the Padua's classification, we could not establish significant difference $(P>0.05)$. Hence, we cannot predict post-operative clinical severity scores and in turn the extent of recovery just by knowing pre-operative CTS severity on pre-operative electrophysiological evaluation.

Similar results were obtained on comparing the difference between other scales used in the present study, namely, visual analogue scale, FSS and DASH score. Significant difference was not found on statistical evaluation $(P>0.05)$.

Schrijver et al. ${ }^{[11]}$ studied 138 patients with completed Levine questionnaire and nerve conduction studies (NCS)

Table 3: Comparison of electrophysiological scores in Padua's modified groups

\begin{tabular}{lccccc}
\hline Group & \multicolumn{5}{c}{ Mean (SD) } \\
\cline { 2 - 5 } & SNAP & Sensory latency $(\boldsymbol{m s})$ & Sensory conduction velocity $(\boldsymbol{m} / \mathbf{s})$ & CMAP $(\boldsymbol{m V})$ & Motor latency \\
\hline Group I & $17.95(5.37)$ & $2.54(0.55)$ & $49.34(7.79)$ & $15.15(1.29)$ & $3.61(0.46)$ \\
Group II & $8.32(6.17)$ & $3.64(1.50)$ & $35.85(6.56)$ & $11.47(4.81)$ & $5.24(2.04)$ \\
Group III & 1.50 & 9.08 & 28.3 & $4.36(3.47)$ & $7.99(3.34)$ \\
\hline
\end{tabular}

SNAP: Sensory nerve action potential, CMAP: Compound muscle action potential, SD: Standard deviation 
Nehete, et al.: A study of various parameters of severity of CTS

Table 4: Comparison of difference between pre- and post-operative scores in Padua's modified groups

\begin{tabular}{lcccc}
\hline Group & \multicolumn{4}{c}{ Score (pre- and post-operative), mean (SD) } \\
\cline { 2 - 5 } & VAS & SSS & FSS & DASH \\
\hline Group I & $6.20(1.48)$ & $23.60(12.97)$ & $14.40(7.96)$ & $47.40(23.10)$ \\
Group II & $6.43(2.46)$ & $22.43(10.03)$ & $17.52(10.52)$ & $47.30(21.69)$ \\
Group III & $6.14(1.77)$ & $21.14(7.80)$ & $16.57(9.16)$ & $51.43(19.51)$ \\
\hline VAS: Visual analogue scale, SSS: Symptom severity score, FSS: functional status score, DASH: Disabilities of arm, shoulder and hand, SD: Standard deviation
\end{tabular}

Table 5: Comparison of preoperative with postoperative scores

\begin{tabular}{lccccc}
\hline Score & Pre-operative & Post-operative & Wilcoxon test & $\boldsymbol{P}$ & Significance \\
\hline VAS score & 7.29 & 0.94 & 5.173 & $<0.001$ & Difference is significant \\
SSS & 25.54 & 3.2 & 5.129 & $<0.001$ & Difference is significant \\
FSS & 18.89 & 2 & 5.022 & $<0.001$ & Difference is significant \\
DASH score & 82.89 & 34.74 & 5.144 & $<0.001$ & Difference is significant
\end{tabular}

VAS: Visual analogue scale, SSS: Symptom severity score, FSS: functional status score, DASH: Disabilities of arm, shoulder and hand

before and after treatment to correlate NCS and clinical outcome measures but could not identify any relationship between pre-operative NCS and the outcome of the surgery, which was also similar to the results of Longstaff et $a .^{[12]}$. Schrijver et al. also studied the relationship between nature or duration of symptoms and severity of electrophysiological impairment. They also concluded that EMG NCS cannot be considered essential in assessing outcome in CTS after surgery.

Khan et al. ${ }^{[13]}$ compared subjective symptoms with psychological factor as well as with electrophysiological severity, and they concluded that subjective symptoms are more correlated with psychological factors than with objective electrophysiological severity of the disease. They even suggested antidepressant drugs in select patients as a treatment apart from analgesics.

\section{CONCLUSIONS}

The aim of the study was to study the various clinical and electrophysiological parameters of severity of CTS and to see if the severity of CTS predicts recovery after surgery. The various clinical severity scores can be effectively used to assess patient's post-operative recovery from symptoms, but these scores fail to predict the extent of recovery the patient will have after surgery just by knowing the pre-operative scores. The difference between pre-operative and post-operative scores is a good indicator of post-operative recovery, but it does not show any correlation when compared with electrophysiological severity. Our study reconfirms the diagnostic role of EMG NCV in CTS, but the electrophysiological severity does not always match the clinical severity. Our study showed that there is no definitive relation between electrophysiological severity and the clinical severity scores.

Hence to summarise, from our study, we can draw the following conclusions:

1. Clinical scores are good to know as a marker of clinical severity of CTS and also as a baseline value to compare with post-operative clinical scores

2. There is no definitive relationship between clinical and electrophysiological severity of CTS

3. Difference between the pre-operative and post-operative clinical severity scores is a good indicator of post-operative recovery

4. Pre-operative scores alone fail to predict the extent of post-operative recovery from the symptoms.

Hence, clinical correlation is the only foolproof way to predict severity and improvement after surgery in CTS-affected patients.

\section{Financial support and sponsorship}

Nil.

\section{Conflicts of interest}

There are no conflicts of interest.

\section{REFERENCES}

1. Bickel KD. Carpal tunnel syndrome. J Hand Surg Am 2010;35:147-52.

2. Levine DW, Simmons BP, Koris MJ, Daltroy LH, Hohl GG, Fossel $\mathrm{AH}$, et al. A self-administered questionnaire for the assessment of severity of symptoms and functional status in carpal tunnel syndrome. J Bone Joint Surg Am 1993;75:1585-92.

3. Simpson JA. Electrical signs in the diagnosis of carpal tunnel and related syndromes. J Neurol Neurosurg Psychiatry 1956;19:275-80.

4. Gilliatt RW, Sears TA. Sensory nerve action potentials in patients with peripheral nerve lesions. J Neurol Neurosurg Psychiatry 1958;21:109-18. 
5. Available from: http://www.wongbakerfaces.org. [Last accessed on 2017 Nov 25].

6. Gummesson C, Atroshi I, Ekdahl C. The disabilities of the arm, shoulder and hand (DASH) outcome questionnaire: Longitudinal construct validity and measuring self-rated health change after surgery. BMC Musculoskelet Disord 2003;4:11.

7. Padua L, LoMonaco M, Gregori B, Valente EM, Padua R, Tonali P, et al. Neurophysiological classification and sensitivity in 500 carpal tunnel syndrome hands. Acta Neurol Scand 1997;96:211-7.

8. El-Hajj T, Tohme R, Sawaya R. Changes in electrophysiological parameters after surgery for the carpal tunnel syndrome. J Clin Neurophysiol 2010;27:224-6.

9. Thatte MR, Mansukhani KA. Compressive neuropathy in the upper limb. Indian J Plast Surg 2011;44:283-97.

10. You H, Simmons Z, Freivalds A, Kothari MJ, Naidu SH.
Relationships between clinical symptom severity scales and nerve conduction measures in carpal tunnel syndrome. Muscle Nerve 1999;22:497-501.

11. Schrijver HM, Gerritsen AA, Strijers $R L$, Uitdehaag $B M$, Scholten RJ, de Vet $\mathrm{HC}$, et al. Correlating nerve conduction studies and clinical outcome measures on carpal tunnel syndrome: Lessons from a randomized controlled trial. J Clin Neurophysiol 2005;22:216-21.

12. Longstaff L, Milner RH, O'Sullivan S, Fawcett P. Carpal tunnel syndrome: The correlation between outcome, symptoms and nerve conduction study findings. J Hand Surg Br 2001;26:475-80.

13. Khan F, Shehna A, Ramesh S, Sandhya KS, Paul R. Subjective symptoms of carpal tunnel syndrome correlate more with psychological factors than electrophysiological severity. Ann Indian Acad Neurol 2017;20:69-72. 